\title{
Spectra-Based Estimates of Certain Time-Domain Parameters of a Bivariate Stationary-Point Process
}

\author{
A. G. RIGAS \\ Department of Electrical Engineering, Demokritos University of Thrace, \\ 67100 Xanthi, Greece
}

Received 19 September 1989; revised 18 October 1990

\begin{abstract}
We consider estimates of certain time-domain parameters of a bivariate stationarypoint process based on modified periodogram statistics. These estimates are shown to be asymptotically normal under regularity conditions. In the computations of the estimates, the advantage of using the FFT algorithm is demonstrated. Three examples, obtained by analyzing two data sets from the field of neurophysiology, are illustrated.
\end{abstract}

\section{INTRODUCTION}

Let $\left\{N_{1}(t), N_{2}(t)\right\},-\infty<t<\infty$, be a bivariate stationary-point process. By $N_{k}(t)$ we denote the number of type- $k$ events that occur in the time interval $[0, t](k=1,2)$.

Before we go on to define certain parameters of the bivariate point process, we make the following assumptions:

(a) The process is strictly stationary.

(b) The process is orderly.

(c) The process satisfies a strong mixing condition.

More details about these assumptions can be found in Brillinger [6] and Cox and Lewis [10].

The first- and second-order properties of a bivariate point process in the time domain can be described by the mean intensities and the second-order product density functions. These functions are defined by

$$
p_{k}=\lim _{h \downarrow 0} \operatorname{Prob}\{\text { a } k \text {-type event occurs in }(t, t+h]\} / h
$$

MATIIEMATICAL BIOSCIENCES 104:185-201 (1991)

CElsevier Science Publishing Co., Inc., 1991

655 Avenue of the Americas, New York, NY 10010

$0025-5564 / 91 / \$ 03.50$ 
and

$$
\begin{array}{r}
p_{k l}(u)=\lim _{h, h^{\prime} \downarrow 0} \operatorname{Prob}\{\text { a } l \text {-type event occurs in }(t+u, t+u+h] \\
\text { and a } \left.k \text {-type event occurs in }\left(t, t+h^{\prime}\right]\right\} / h h^{\prime},
\end{array}
$$

where $k, l=1,2$. Relations (1) and (2) hold if assumptions (a) and (b) are valid. In relation to these functions we can define the cumulant densities as

$$
q_{k l}(u)=\rho_{k l}(u)-p_{k} p_{l}, \quad u \neq 0 .
$$

Assumption (c) ensures that

$$
p_{k l}(u) \rightarrow p_{k} p_{l} \quad \text { and } \quad q_{k l}(u) \rightarrow 0 \quad \text { as }|u| \rightarrow \infty .
$$

In the frequency domain, the second-order properties of the bivariate point process are described by the spectra. These functions are defined by

$$
\begin{array}{r}
g_{k l}(\lambda)=(2 \pi)^{-1} p_{k} \delta\{k-1\}+(2 \pi)^{-1} \int_{-\infty}^{+\infty} q_{k l}(u) \exp (-i \lambda u) d u, \\
-\infty<\lambda<\infty,
\end{array}
$$

provided that

$$
\int_{-\infty}^{+\infty}\left|q_{k l}(u)\right| d u<\infty, \quad k, l=1,2 .
$$

The function $\delta\{\}$ is the Kronecker delta given by

$$
\delta\{k-l\}=\left\{\begin{array}{cc}
1, & k=l \\
0 & k \neq l .
\end{array}\right.
$$

In practice, however, since the cumulant density $q_{k l}(u)$ is calculated at intervals of length $b$, expression (1) is approximated by

$$
f_{k l}(\lambda)=(2 \pi)^{-1} p_{k} \delta\{k-l\}+(2 \pi)^{-1} b \sum_{j=-\infty}^{+\infty} q_{k l}\left(u_{j}\right) \exp \left(-i \lambda u_{j}\right),
$$

where $u_{j}=b j, j=0, \pm 1, \pm 2, \ldots$. The value of $b$ is assumed to be small and is chosen in such a way that $b T$ is constant, where $T$ is the length of the interval in which the events of the bivariate point process are observed. For more details about this, refer to Brillinger [7] and Doss [11].

Expression (3) generalizes the definition of the power spectrum of a univariate point process $(k=l)$ and the definition of the cross-spectrum of a bivariate point process $(k \neq l)$. Details about these definitions are given in Bartlett [1], Brillinger [5], and Cox and Lewis [10]. 
The inverse expressions of (3) and (4) are

$$
q_{k l}(u)=\int_{-\infty}^{+\infty}\left(g_{k l}(\lambda)-\frac{p_{k}}{2 \pi} \delta\{k-l\}\right) \exp (i \lambda u) d \lambda
$$

and

$$
q_{k l}\left(u_{j}\right)=\int_{-\pi / b}^{+\pi / b}\left(f_{k l}(\lambda)-\frac{p_{k}}{2 \pi} \delta\{k-l\}\right) \exp \left(i \lambda u_{j}\right) d \lambda
$$

Using expressions (3)-(6), we obtain

$$
f_{k l}(\lambda)-\frac{p_{k}}{2 \pi} \delta\{k-l\}=\sum_{j=-\infty}^{+\infty}\left[g_{k l}\left(\lambda+\frac{2 \pi j}{b}\right)-\frac{p_{k}}{2 \pi} \delta\{k-l\}\right] .
$$

The frequencies $\lambda+2 \pi j / b,-\lambda+2 \pi j / b, j=0, \pm 1, \pm 2, \ldots$, are called the aliases of the frequency $\lambda$ (see Tukey [17]). In the case that $g_{k l}(\lambda)$ behaves as $\left(p_{k} / 2 \pi\right) \delta\{k-l\}$ for $|\lambda|>\pi / b$, it follows from (7) that

$$
f_{k l}(\lambda) \approx g_{k l}(\lambda)
$$

A function of interest is the second-order intensity function defined by

$$
m_{k l}(u)=\frac{q_{k l}(u)}{p_{k}}+p_{l}, \quad u \neq 0
$$

This is a conditional probability interpreted as

$$
\lim _{h \downarrow 0} \operatorname{Prob}\{l \text {-type event in }(t, t+h] \mid k \text {-type event at } t\} \text {. }
$$

It is a common procedure for researchers in point processes to consider the square root of the second-order intensity function in order to stabilize the variance (see Brillinger [7]).

We shall concentrate on estimating the function $m_{k l}(u)$ and its square root by using methods of the frequency domain.

\section{ESTIMATES OF TIME-DOMAIN PARAMETERS}

Before we go on to consider an estimate of the $m_{k l}(u)$, it is necessary to obtain an estimate of $q_{k l}(u)$.

As we have mentioned previously, the estimate of $q_{k l}(u)$ will be based on the modified periodogram statistic. This statistic is defined by

$$
\tilde{I}_{k l}^{(T)}(\lambda)=\frac{1}{2 \pi T} \tilde{d}_{k}^{(T)}(\lambda) \overline{\tilde{d}^{(T)}(\lambda)}
$$


where $\tilde{d}_{k}^{(T)}(\lambda)$ is the finite Fourier-Stieltjes transform on the interval $[0, T]$ given by

$$
\tilde{d}_{k}^{(T)}(\lambda)=\int_{0}^{T} \exp (i \lambda t)\left\{d N_{k}(t)-\tilde{p}_{k} d t\right\}
$$

We denote the conjugate function of $\tilde{d}_{l}^{(T)}(\lambda)$ by $\overline{\tilde{d}_{l}^{(T)}(\lambda)}$ and the estimate of the mean intensity of the point process $\left.N_{k}(t), k=1,2\right)$, by $\tilde{p}_{k}=N_{k}(T) / T$. These functions are considered by Tuan [16] and Rigas [14].

To improve the properties of our estimate, we insert data windows into the finite Fourier-Stieltjes transform as follows:

$$
\hat{d}_{k}^{(T)}(\lambda)=\int_{0}^{T} h_{k}^{(T)}(t) \exp (i \lambda t)\left\{d N_{k}(t)-\hat{p}_{k} d t\right\}
$$

where $\hat{p}_{k}=d_{k}^{(T)}(0) / H_{k}^{(T)}(0)$ is a modified estimate of the mean intensity of $N_{k}(t), k=1,2$. The use of a data window in (11) reduces considerably the phenomenon of leakage (see Bloomfield [2, p. 80]) and hence improves the characteristics of the estimate. The quantities $d_{k}^{(T)}(0), H_{k}^{(T)}(0)$ are given by

$$
d_{k}^{(T)}(0)=\int_{0}^{T} h_{k}^{(T)}(t) d N(t)
$$

and

$$
H_{k}^{(T)}(0)=\int_{0}^{T} h_{k}^{(T)}(t) d t=T \int_{0}^{1} h_{k}(t) d t
$$

where $h_{k}(t), 0<t<\infty$, is a bounded function of bounded variation and vanishes for $t>1$ (see Brillinger [8, p. 91]).

The modified periodogram statistic now takes the form

$$
\hat{I}_{k l}^{(T)}(\lambda)=\left\{2 \pi H_{k l}^{(T)}(0)\right\}^{-1} \hat{d}_{k}^{(T)}(\lambda){\overline{d_{l}^{(T)}(\lambda)}}
$$

where

$$
H_{k l}^{(T)}(0)=\int_{0}^{T} h_{k}^{(T)}(t) h_{l}^{(T)}(t) d t=T \int_{0}^{1} h_{k}(t) h_{l}(t) d t .
$$

More details about data windows can be found in Brillinger [8].

In practice, the finite Fourier-Stieltjes transform can be approximated by

$$
\hat{d}_{k}^{(T)}(\lambda) \approx \sum_{t=0}^{T-1} h_{k}^{(T)}(t) \exp (i \lambda t)\left[\left\{N_{k}(t+1)-N_{k}(t)\right\}-\hat{p}_{k}\right]
$$


This approximation allows the use of the FFT algorithm in the computation of $\hat{d}_{k}^{(T)}(\lambda)$. Such an approach has been introduced by Rigas [14] in the estimation of the power spectrum of a univariate stationary-point process. The sampling time interval is taken to be 1 . This ensures that the sampled process is orderly, because the smallest distance between spikes is $1 \mathrm{~ms}$.

The estimate of $q_{k l}(u)$ is now given by

$$
\hat{q}_{k /}(u)=\int_{-\pi / b}^{+\pi / b}\left(\hat{I}_{k I}^{(T)}(\lambda)-\frac{\hat{p}_{k}}{2 \pi} \delta\{k-l\}\right) \exp (i \lambda u) d \lambda
$$

and hence

$$
\hat{m}_{k l}(u)=\frac{\hat{q}_{k l}(u)}{\hat{p}_{k}}+\hat{p}_{l}, \quad u \neq 0
$$

The properties of $\hat{m}_{k l}(u)$ in the time domain have been examined by Brillinger [7] and Cox [9].

\section{ASYMPTOTIC RESULTS}

In what follows we assume that higher-order joint cumulant densities of the bivariate stationary-point process can be written as

$$
\begin{gathered}
\operatorname{cum}\left\{d N_{k_{1}}\left(t+u_{1}\right), \ldots, d N_{k_{j-1}}\left(t+u_{j-1}\right), d N_{k_{j}}(t)\right\} \\
=q_{k_{1}, \ldots, k_{j}}\left(u_{1}, \ldots, u_{j-1}\right) d u_{1} \cdots d u_{j-1} d t
\end{gathered}
$$

for $k_{1}, \ldots, k_{j}=1,2$ and $j=1,2,3, \ldots$ The arguments $u_{1}, u_{2}, \ldots, u_{j-1}$ are distinct for $j=2,3, \ldots$.

ASSUMPTION 1

The stationary point process $\left\{N_{1}(t), N_{2}(t)\right\},-\infty<t<\infty$, possesses moments of all orders and is such that

$$
\int \cdots \int\left(1+\left|u_{l}\right|\right)\left|q_{k_{1}, \ldots, k_{j}}\left(u_{1}, \ldots, u_{j-1}\right)\right| d u_{1} \cdots d u_{j-1}<\infty
$$

for $k_{1}, \ldots, k_{j}=1,2 ; l=1,2, \ldots, j-1$, and $j=2,3, \ldots$.

This assumption has been considered in Brillinger [5] and is valid in the case that the bivariate process is strictly stationary. 


\section{THEOREM 1}

Let $\left\{N_{1}(t), N_{2}(t)\right\},-\infty<t<\infty$, be a bivariate point process satisfying $A s$ sumption 1. Suppose that the estimate $\hat{q}_{k l}(u)$ is given by (15). Then

$$
E\left\{\hat{q}_{k l}(u)\right\}=q_{k l}(u)+O\left(T^{-1}\right),
$$

$\operatorname{cov}\left\{\hat{q}_{k_{1} l_{1}}(u), \hat{q}_{k_{2} l_{2}}(v)\right\}$

$$
\begin{aligned}
=2 \pi \frac{H_{k_{1} l_{1} k_{2} l_{2}}^{(T)}(0)}{H_{k_{1} l_{1}}^{(T)}(0) H_{k_{2} l_{2}}^{(T)}(0)}\left[\int_{-\pi / b}^{+\pi / b} f_{k_{1} l_{2}}(\lambda) f_{l_{1} k_{2}}(-\lambda) \exp \{i \lambda(u+v)\} d \lambda\right. \\
+\int_{-\pi / b}^{+\pi / b} f_{k_{1} k_{2}}(\lambda) f_{l_{1} l_{2}}(-\lambda) \exp \{i \lambda(u-v)\} d \lambda \\
+\int_{-\pi / b}^{+\pi / b} \int_{-\pi / b}^{+\pi / b} f_{k_{1} l_{1} k_{2} l_{2}}(\lambda,-\lambda,-\mu) \\
\quad \times \exp \{i(\lambda u+\mu v)\} d \lambda d \mu] \\
+O\left(T^{-2}\right), \quad \text { for } u, v \neq 0,
\end{aligned}
$$

where

$$
\begin{aligned}
H_{l_{1} l_{1} k_{2} l_{2}}^{(T)}(0) & =\int_{0}^{T} h_{k_{1}}^{(T)}(t) h_{l_{1}}^{(T)}(t) h_{k_{2}}^{(T)}(t) h_{l_{2}}^{(T)}(t) d t \\
& =T \int_{0}^{1} h_{k_{1}}(t) h_{l_{1}}(t) h_{k_{2}}(t) h_{l_{2}}(t) d t
\end{aligned}
$$

Furthermore, $\hat{q}_{k l}(u)$ is asymptotically normal with the above first- and second-order moment structure.

We are now in a position to develop the asymptotic properties of the estimate $\hat{m}_{k l}(u)$.

\section{THEOREM 2}

Let $\left\{N_{1}(t), N_{2}(t)\right\},-\infty<t<\infty$, be a bivariate stationary-point process satisfying Assumption 1. Suppose that the estimate $\hat{m}_{k l}(u)$ is given by (16). Then $\hat{m}_{k l}(u)$ is asymptotically normal with first-and second-order moments 
given by

$$
\begin{aligned}
& E\left\{\hat{m}_{k l}(u)\right\}=m_{k l}(u)+O\left(T^{-1}\right), \\
& \operatorname{cov}\left\{\hat{m}_{k_{1} l_{1}}(u), \hat{m}_{k_{2} l_{2}}(v)\right\} \\
& =\frac{1}{p_{k_{1}} p_{k_{2}}} \operatorname{cov}\left\{\hat{q}_{k_{1} l_{1}}(u), \hat{q}_{k_{2} l_{2}}(v)\right\} \\
& -\frac{q_{k_{2} l_{2}}(v)}{p_{k_{1}} p_{k_{2}}^{2}}\left(\frac{2 \pi H_{k_{1} l_{1} k_{2}}^{(T)}(0)}{H_{k_{1} l_{1}}^{(T)}(0) H_{k_{2}}^{(T)}(0)}\right) \int_{-\pi / b}^{+\pi / b} f_{k_{1} l_{1} k_{2}}(\lambda,-\lambda) \exp (i \lambda u) d \lambda \\
& +\frac{1}{p_{k_{1}}}\left(\frac{2 \pi H_{k_{1} l_{1} k_{2}}^{(T)}(0)}{H_{k_{1} l_{1}}^{(T)}(0) H_{k_{2}}^{(T)}(0)}\right) \int_{-\pi / b}^{+\pi / b} f_{k_{1} l_{1} l_{2}}(\lambda,-\lambda) \exp (i \lambda u) d \lambda \\
& -\frac{q_{k_{2} l_{2}}(u)}{p_{k_{1}}^{2} p_{k_{2}}}\left(\frac{2 \pi H_{k_{1} k_{2} l_{2}}^{(T)}(0)}{H_{k_{1}}^{(T)}(0) H_{k_{2} l_{2}}^{(T)}(0)}\right) \int_{-\pi / b}^{+\pi / b} f_{k_{1} k_{2} l_{2}}(0, \lambda) \exp (i \lambda v) d \lambda \\
& +\frac{1}{p_{k_{2}}}\left(\frac{2 \pi H_{l_{1} k_{2} l_{2}}^{(T)}(0)}{H_{l_{1}}^{(T)}(0) H_{k_{2} l_{2}}^{(T)}(0)}\right) \int_{-\pi / b}^{+\pi / b} f_{l_{1} k_{2} l_{2}}(0, \lambda) \exp (i \lambda v) d \lambda \\
& -\frac{q_{k_{1} l_{1}}(u)}{p_{k_{1}}^{2}}\left(\frac{2 \pi H_{k_{1} l_{2}}^{(T)}(0)}{H_{k_{1}}^{(T)}(0) H_{l_{2}}^{(T)}(0)}\right) f_{k_{1} l_{2}}(0) \\
& -\frac{q_{k_{2} l_{2}}(v)}{p_{k_{2}}^{2}}\left(\frac{2 \pi H_{k_{1} l_{2}}^{(I)}(0)}{H_{l_{1}}^{(T)}(0) H_{k_{2}}^{(T)}(0)}\right) f_{l_{1} k_{2}}(0) \\
& +\frac{2 \pi H_{l_{1} l_{2}}^{(T)}(0)}{H_{l_{1}}^{(T)}(0) H_{l_{2}}^{(T)}(0)} f_{l_{1} l_{2}}(0) \\
& +\frac{q_{k_{1} l_{1}}(u) q_{k_{2} l_{2}}(v)}{p_{k_{1}}^{2} p_{k_{2}}^{2}}\left(\frac{2 \pi H_{k_{1} k_{2}}^{(T)}(0)}{H_{k_{1}}^{(T)}(0) H_{k_{2}}^{(T)}(0)}\right) f_{k_{1} k_{2}}(0)+O\left(T^{-2}\right) \text {, } \\
& \text { for } u, v \neq 0 \text {, }
\end{aligned}
$$

where $\operatorname{cov}\left\{\hat{q}_{k_{1} l_{1}}(u), \hat{q}_{k_{2} l_{2}}(v)\right\}$ is obtained from (17).

We are also interested in finding the properties of the square root of $\hat{m}_{k l}(u)$. This is done in the following corollary.

\section{COROLLARY 1}

Let $\left\{N_{1}(t), N_{2}(t)\right\},-\infty<t<\infty$, be a bivariate stationary-point process satisfying Assumption 1. Suppose that $\hat{\xi}_{k l}(u)-\sqrt{\hat{m}_{k l}(u)}$ is an estirnate of the square root of $m_{k l}(u)$. Then $\hat{\xi}_{k l}(u)$ is asymptotically normal with the first-and 
second-order moments given by

$$
\begin{gathered}
E\left\{\hat{\xi}_{k l}(u)\right\}=\xi_{k l}(u)+O\left(T^{-1}\right), \\
\operatorname{cov}\left\{\hat{\xi}_{k_{1} l_{1}}(u), \hat{\xi}_{k_{2} l_{2}}(v)\right\}=\frac{1}{4 \xi_{k_{1} l_{1}}(u) \xi_{k_{2} l_{2}}(v)} \operatorname{cov}\left\{\hat{m}_{k_{1} l_{l}}(u), \hat{m}_{k_{2} l_{2}}(v)\right\}
\end{gathered}
$$

where $\operatorname{cov}\left\{\hat{m}_{k_{1} l_{1}}(u), \hat{m}_{k_{2} l_{2}}(v)\right\}$ is obtained from (18). Relation (19) holds provided that $\xi_{k_{1} l_{1}}(u)$ and $\xi_{k_{2} l_{2}}(v)$ are different from zero for every $u$ and $v$.

The proofs of the theorems and the corollary are discussed in the appendix of the paper.

\section{MUSCLE SPINDLES AND EXPERIMENTAL METHODS}

Muscle spindles are one particularly important class of muscle receptors that are thought to have an important role in the initiation of movement and the maintenance of posture. The muscle spindle is a transducer that responds to different stimuli. Muscle spindles consist of parts of the "skeletal" muscles that are concerned with posture or movement. Most "skeletal" muscles contain a number of these receptors, which lie in parallel with the extrafusal fibers. They consist of a number of specialized muscle fibers lying parallel to each other and partially contained within a fluid-filled capsule of connective tissue (see Boyd [3]). The fibers within a muscle spindle, known as intrafusal fibers, are considerably shorter than the extrafusal muscle fibers.

The effects of the imposed stimuli on the intrafusal muscle fibers are transmitted to the spinal cord by the axons of sensory nerves closely associated with the muscle spindle. The terminal branches of the sensory axons form spirals around the central region of the intrafusal muscle fibers.

When a muscle is held at a fixed length, the sensory axons from the muscle spindle produce nerve impulses at a constant rate that depends upon the muscle length. The nerve impulse is a localized voltage change that occurs across the membrane surrounding the nerve cell and axon. Its amplitude is approximately $100 \mathrm{mV}$, and its duration $1 \mathrm{~ms}$. Nerve impulses are known as "action potentials" or, because of their relatively short duration, "spikes."

In addition to sensory nerves associated with the muscle spindle, the intrafusal muscle fibers are innervated by the axons of a group of cells lying within the spinal cord. These cells are called gamma-motoneurons or fusimotor neurons and innervate only intrafusal muscle fibers. When a gamma-motoneuron affects the muscle spindle by transmitting nerve impulses to the intrafusal muscle fibers (gamma stimulation), the response of the muscle spindle sensory axons (called the Ia response) is modified. It has 
been suggested that activity in the gamma-motoneuron axons may modify the mechanical properties of the intrafusal fibers or exert a direct effect on the mechanisms involved in the conversion of mechanical strain in the intrafusal fibers to discharge of nerve impulses in the sensory axon. For more details about the muscle spindle and its properties, refer to Boyd [4] and Matthews [13].

The tenuissimus muscle in anesthetized cats was used in the experiments, and the responses of single sensory axons in dorsal root filaments were recorded. The axons of fusimotor neurons $\left(\gamma_{s}\right)$ were stimulated by sequences of pulses at twice threshold having an exponential distribution of intervals. By "threshold" we mean the critical value over which a nerve impulse will occur. Fifteen seconds of responses were recorded when (1) no stimulation was present and (2) a gamma stimulation was present, with the tenuissimus muscle held at a fixed length. The times of occurrence of the spikes of the sensory axons and the stimulus pulses were measured and stored in computer files.

\section{EXAMPLES}

In this section we present the estimates of the autointensity functions and the cross-intensity function obtaincd by analyzing the two data scts that are illustrated below.

Figure 1 describes the Ia response of the muscle spindle when no stimulation is present. In Figure 1A the histogram of the intervals between spikes of the Ia response is shown. The value of $h$ was taken to be $2 \mathrm{~ms}$. Figure 1B shows the scatter diagram of adjacent intervals between spikes of the Ia response, whereas Figure $1 \mathrm{C}$ presents a sequence of nerve impulses of the la response.

Figure 2 describes the Ia response of the muscle spindle when a gamma stimulation is present. The graphs shown in Figures $2 \mathrm{~A}-\mathrm{C}$ are similar with those discussed in Figures 1A-C.

The data sets consist of 538 nerve impulses that occurred irregularly in an interval of length $T=15,872 \mathrm{~ms}$. In order to be able to use the FFT algorithm we work in the way described below.

Three steps are taken in the computation of the modified discrete Fourier-Stieltjes transform.

Step 1. The quantities $\left[\left\{N_{k}(t+1)-N_{k}(t)\right\}-\hat{p}_{k}\right], t=0,1, \ldots, T-1$, are calculated, where $\hat{p}_{k}$ is the estimate of the mean intensity of the point process $N_{k}(t), k=1,2$.

Step 2. We multiply the quantities calculated in step 1 by a split bell cosine window (see Bloomfield [2]) with a $10 \%$ tapering at the beginning and end of the data. 

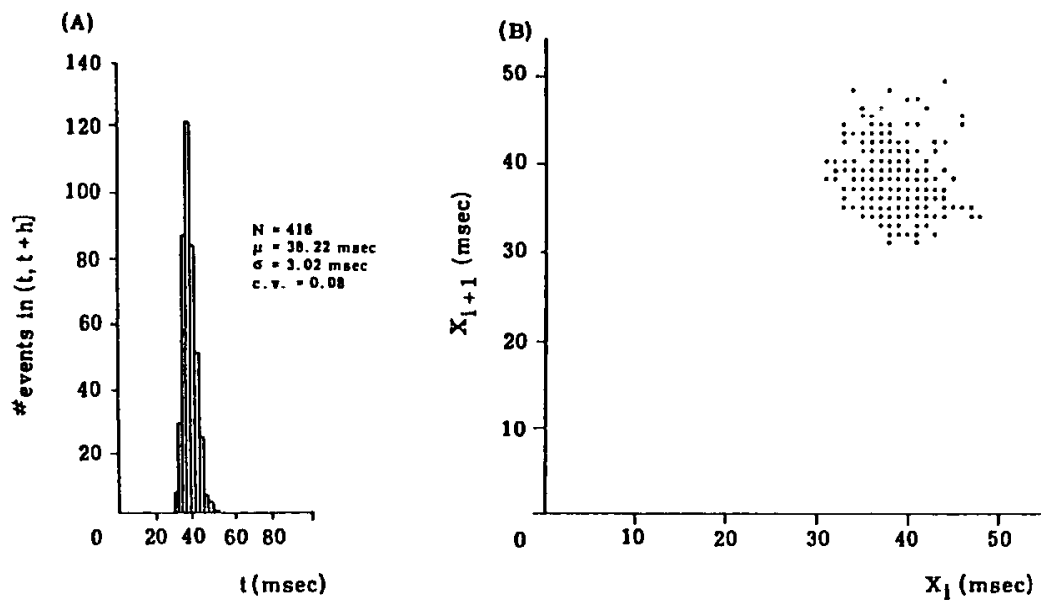

(C)

Ia

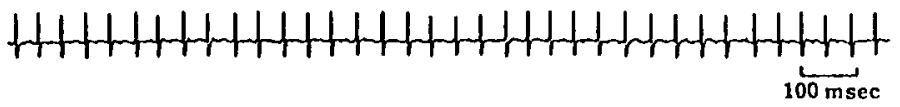

FIG. 1. Ia response when no stimulation is present. (A) Histogram of the intcrspikc intervals of the Ia response; (B) scatter diagram of adjacent intervals between spikes of the Ia response; $(C)$ sequence of spikes of the Ia response.

Step 3. We extend the record length from $T=15,872$ to $S=16,384$ by adding 512 zeroes to the end of the tapered data. By adding zeroes we get a power of 2, and thus we are able to use the Radix-2 FFT algorithm.

The modified periodogram statistic can now be computed from Equation (12). Then the estimate $\dot{m}_{k l}(u)$ is calculated by approximating (15) with the expression

$$
\hat{q}_{k l}(u) \approx \sum_{s=1}^{Q}\left[\hat{I}_{k l}^{(T)}\left(\frac{2 \pi s}{Q}\right)-\frac{\hat{p}_{k}}{2 \pi} \delta\{k-l\}\right] \exp \left(i \frac{2 \pi s u}{Q}\right),
$$

where $Q=S / b$. In our computations we take $b=1$ and $u=0, \pm 1, \pm 2, \ldots$.

In the case of a univariate point process $N_{2}(t)$ and under the assumption that this process is Poisson, the estimate $\hat{m}_{22}(u)$ is approximately normal with mean $p_{2}$ and variance

$$
(b T)^{-1} \frac{\int h^{4}(t) d t}{\left(\int h^{2}(t) d t\right)^{2}}, \quad u \neq 0 .
$$



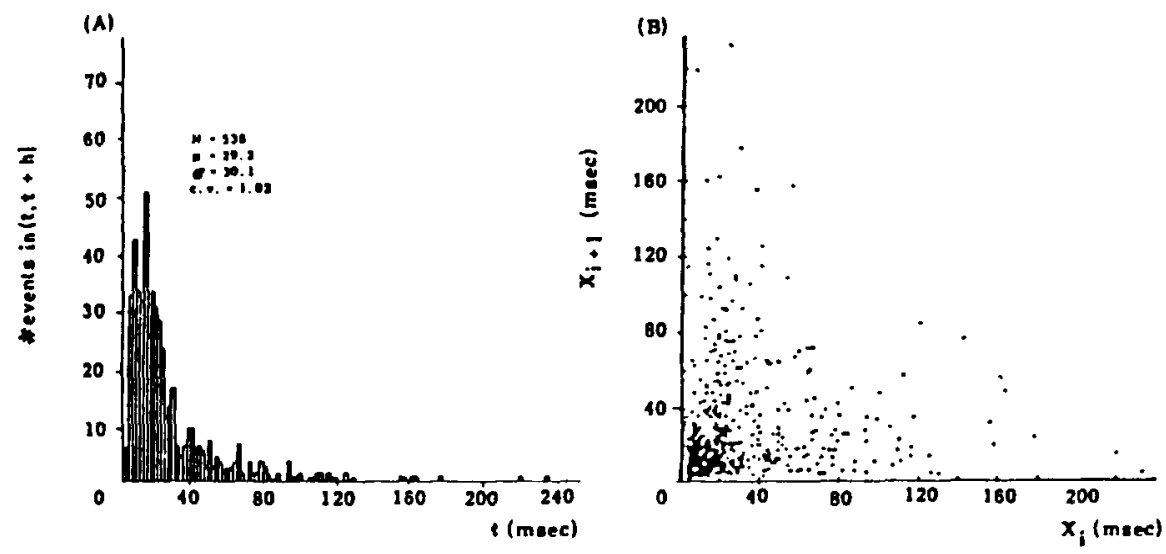

(c)

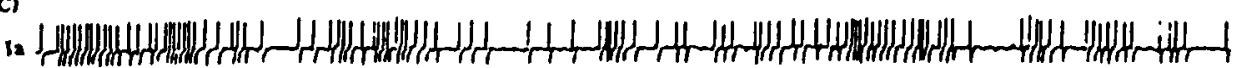

\section{г}

FIG. 2. Ia response when a gamma stimulation is present. (A) Histogram of interspike intervals of the Ia response; (B) scatter diagram of adjacent intervals between spikes of the Ia response; (C) sequence of spikes of the Ia response when a gamma stimulation $\left(\gamma_{\mathrm{s}}\right)$ is present.

This result follows from Theorem 2, because the power spectrum of $N_{2}(t)$ is equal to $p_{2} / 2 \pi$. For a split bell cosine the quantity $\left(\int h^{4}(t) d t\right) /\left(\int h^{2}(t) d t\right)^{2}$ is equal to 1.116 (see Bloomfield [2]). The asymptotic variance of $\hat{m}_{22}(u)$, under the assumption that $N_{2}(t)$ is a Poisson point process, is in agreement with Brillinger's result (see Brillinger [7]).

Figure 3A shows the estimate of the autointensity function of the spontaneous Ia response of the muscle spindle. This is the case where no stimulation was applied to the muscle spindle. The number of events observed in this case was $N_{2}(T)=416$. The extreme regularity of the response is quite clear. The increments of the process become uncorrelated more than $0.5 \mathrm{~s}$ apart, because the process behaves in some sense as a Poisson process. The estimate is zero for the first $32 \mathrm{~ms}$ because no pulses occurred closer together than that interval. The estimate of the mean interval between pulses is $1 / \hat{p}_{2}=0.038 \mathrm{~s}$ and is exactly the point at which the first maximum occurs. The other maxima occur at multiples of this 


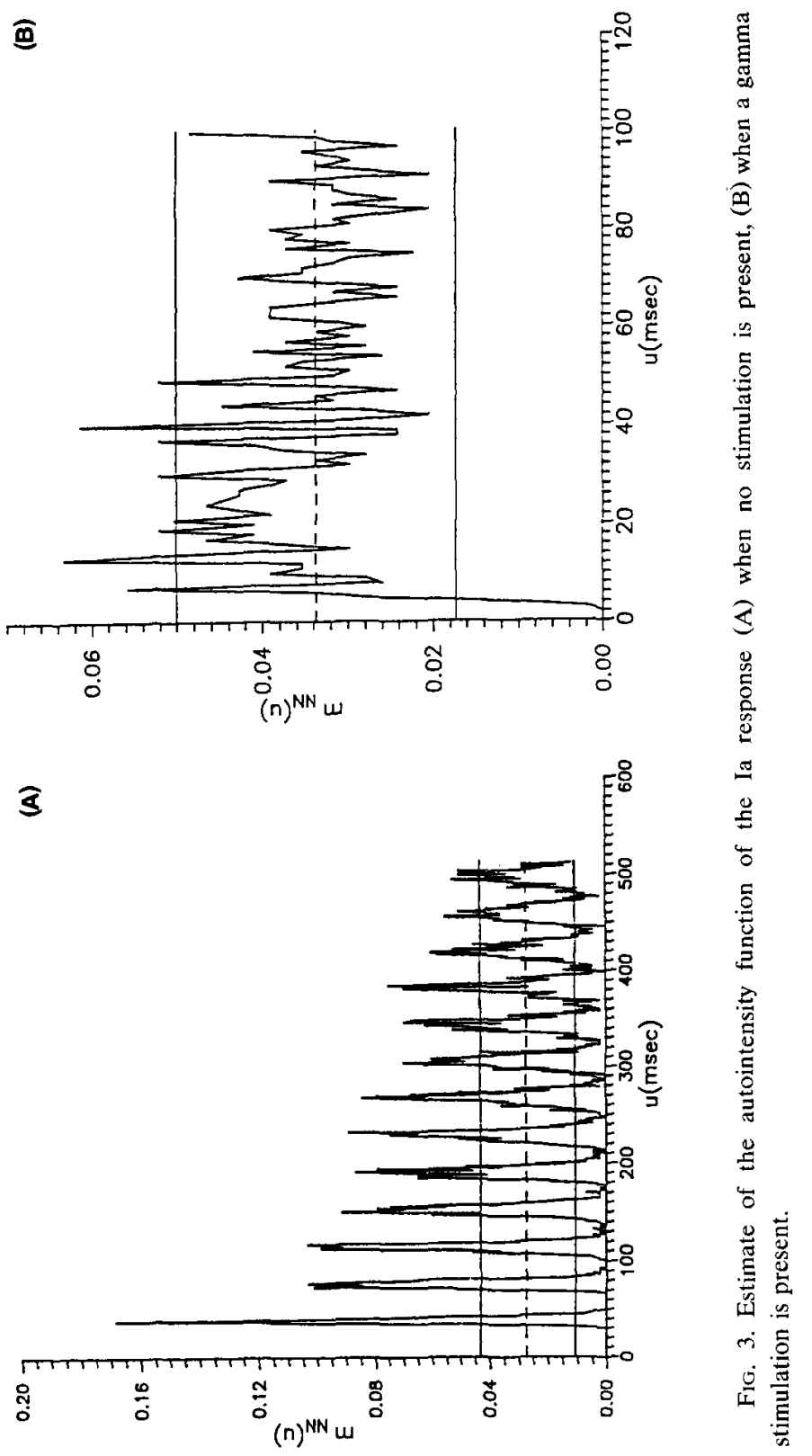




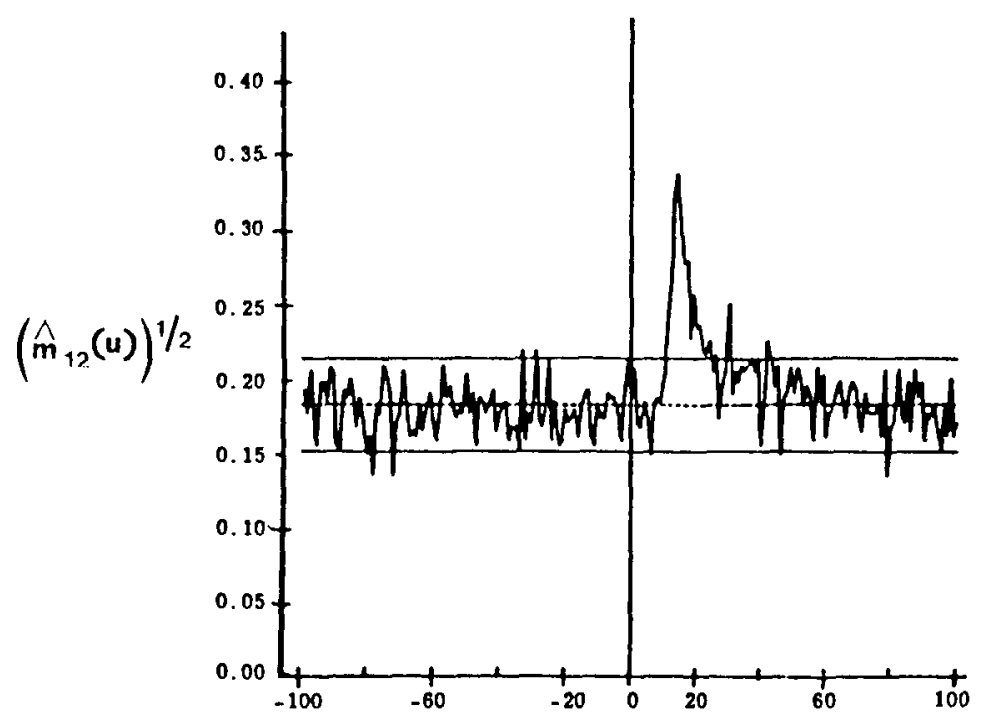

Fig. 4. Square root of the estimate of the cross-intensity function when a gamma stimulation is imposed on the muscle spindle.

value. The dashed line corresponds to $\hat{p}_{2}$, and the solid horizontal lines correspond to $\hat{p}_{2} \pm 2.07(b T)^{-1 / 2}$. Values of the estimate outside the solid horizontal lines indicate deviations from a Poisson process, which is a completely random process.

Figure 3B shows the estimate of the autointensity function of the Ia response when a gamma stimulation is applied to the muscle spindle. It is clear that the presence of the stimulus destroys completely the regularity of the Ia response. Another characteristic of the estimate is the depression near the origin. After that the estimate becomes a constant, which implies that the Ia response behaves in some sense as a Poisson point process. The number of events observed in this case was $N_{2}(T)=538$. The dashed and horizontal lines are obtained in the same way as in Fig. 3A. We did not use the square root of the estimate of the autointensity function, because in both cases described above, the estimate was zero for some values of $u$.

Figure 4 shows the square root of the estimate of the cross-intensity function of the Ia response when a gamma stimulation is present. The number of events of the stimulus applied to the muscle spindle was $N_{1}(T)=1010$. The effect of gamma stimulation is clear when the distance between a Ia pulse and a gamma pulse is in the interval $10-30 \mathrm{~ms}$. It follows from Corollary 1 that the estimate $\sqrt{\hat{m}_{12}(u)}$ is approximately 
normal with mean $\sqrt{p_{2}}$ and variance

$$
\frac{2 \pi}{4 p_{1}^{2} p_{2} T} \frac{\int h^{4}(t) d t}{\left(\int h^{2}(t) d t\right)^{2}} \int_{-\pi / b}^{+\pi / b} f_{11}(\lambda) f_{22}(\lambda) d \lambda, \quad u \neq 0 .
$$

This result holds under the assumption that the two processes are independent.

Furthermore, if the two univariate point processes are Poisson, the asymptotic variance becomes

$$
\left(4 h p_{1} T\right)^{-1} \frac{\int h^{4}(t) d t}{\left(\int h^{2}(t) d t\right)^{2}} .
$$

This result follows from (21) because for Poisson point processes we have $f_{11}(\lambda)=p_{1} / 2 \pi$ and $f_{22}(\lambda)=p_{2} / 2 \pi$.

The dashed line in Figure 4 corresponds to $\sqrt{\hat{p}_{2}}$, and the solid horizontal lines correspond to

$$
\sqrt{\hat{p}_{2}} \pm \frac{1.056 \pi}{\hat{p}_{1}\left(b \hat{p}_{2} T\right)^{1 / 2}}\left[\frac{1}{Q} \sum_{s=1}^{Q-1} \hat{I}_{11}^{(T)}\left(\frac{2 \pi s}{Q}\right) \hat{I}_{22}^{(T)}\left(\frac{2 \pi s}{\bar{Q}}\right)\right]^{1 / 2}
$$

This approximate confidence interval follows from (21). In our computations we take $b=1$ and $Q=S$. Values of the estimate outside the solid horizontal lines indicate deviations from independence between the two point processes.

\section{CONCLUSIONS}

The use of methods of the frequency domain in the estimation of certain time-domain parameters has been demonstrated in this work. This approach has the advantage of employing the FFT algorithm in the computation of the estimates.

In Table 1, we compare the times required for the computation of the square root of the estimate of the cross-intensity function when a frequency-domain (FD) approach is used by taking advantage of the FFT

TABLE 1

\begin{tabular}{rccc}
\hline$N_{1}(T)$ & $N_{2}(T)$ & FD & TD \\
\hline 538 & 1010 & $14 \mathrm{~s}$ & $15 \mathrm{~s}$ \\
750 & 1500 & $14 \mathrm{~s}$ & $32 \mathrm{~s}$ \\
1000 & 2000 & $14 \mathrm{~s}$ & $58 \mathrm{~s}$ \\
\hline
\end{tabular}


algorithm and when a direct time-domain (TD) approach based on relation (4) is used.

The computations were carried out with a VAX/8350 computer. It is obvious that the FD approach becomes more efficient as the number of events of the input and output processes in the interval $(0, T]$ increases. The gain in computing time will be greater when the computations of the estimate are carried out with a small personal computer.

The reduction in computing time will become more obvious in the case of estimating higher-order parameters of the bivariate point process in both time and frequency domains. We hope that this approach will be applicable in other areas of research as well.

I would like to thank Professor G. P. Moore and Dr. J. R. Rosenberg for providing the data sets for this analysis. I also would like to thank Dr. J. R. Rosenberg for many helpful discussions and two anonymous referees for their constructive comments, which led to the presentation of this paper.

\section{APPENDIX: PROOFS OF THEOREMS}

PROOF OF THEOREM 1

Following Theorem 4.1 of Brillinger [5], we get

$$
E\left\{\hat{d}_{k}^{(T)}(\lambda) \hat{d}_{l}^{(T)}(-\lambda)\right\}=2 \pi H_{k l}^{(T)}(0) f_{k l}(\lambda)+O(1)
$$

and

$$
\begin{aligned}
& \operatorname{cov}\left\{\hat{d}_{k_{1}}^{(T)}(\lambda) \hat{d}_{l_{1}}^{(T)}(-\lambda), \hat{d}_{k_{2}}^{(T)}(\mu) \hat{d}_{2}^{T)}(-\mu)\right\} \\
&=(2 \pi)^{2} H_{k_{1} l_{1} k_{2} l_{2}}^{(T)}(0) f_{k_{1} l_{1} k_{2} l_{2}}^{(T)}(\lambda,-\lambda,-\mu)+O(1) \\
&+2 \pi H_{k_{1} k_{2}}^{(T)}(\lambda-\mu) H_{l_{1} l_{2}}^{(T)}(-\lambda+\mu) f_{k_{1} k_{2}}(\lambda) f_{l_{1} l_{2}}(-\lambda)+O(1) \\
&+2 \pi H_{k_{1} l_{2}}^{(T)}(\lambda+\mu) H_{l_{1} k_{2}}^{(T)}(-\lambda-\mu) f_{k_{1} l_{2}}(\lambda) f_{l_{1} k_{2}}(-\lambda)+O(1) .
\end{aligned}
$$

The required results for the first- and second-order moments follow from the above expressions and the fact that

$$
\int_{-\pi / b}^{+\pi / b} H_{k_{1} k_{2}}^{(T)}(\lambda-\mu) H_{l_{1} l_{2}}^{(T)}(-\lambda+\mu) e^{-i \mu v} d \mu=2 \pi e^{-i \lambda v} H_{k_{1} l_{1} k_{2} l_{2}}^{(T)}(0)+O(1)
$$

The asymptotic normality follows from the fact that

$$
T^{J / 2} \operatorname{cum}\left\{\hat{q}_{k_{1} l_{1}}\left(u_{1}\right), \ldots, \hat{q}_{k_{J} l_{J}}\left(u_{J}\right)\right\} \rightarrow 0 \text { as } T \rightarrow \infty \text { and } J>2,
$$


since

$$
\operatorname{cum}\left\{\hat{I}_{k_{1} l_{1}}^{(T)}\left(\lambda_{1}\right), \ldots, \hat{I}_{k_{J}}^{(T)} l_{l}\left(\lambda_{J}\right)\right\}-O\left(T^{-J+1}\right)
$$

A proof of this result can be found in Brillinger [8] in the case of ordinary time series. A similar proof holds for point processes.

\section{PROOF OF THEOREM 2}

From the results of Torres-Melo [15] and Lemma 3 of Mann and Wald [12], we have

$$
\begin{aligned}
\hat{m}_{k l}(u)-m_{k l}(u)= & \frac{1}{p_{k}}\left\{\hat{q}_{k l}(u)-q_{k l}(u)\right\}-\frac{q_{k l}(u)}{p_{k}^{2}}\left(\hat{p}_{k}-p_{k}\right) \\
& +\left(\hat{p}_{l}-p_{l}\right)+o_{p}\left(T^{-1}\right) .
\end{aligned}
$$

The first-order moment of $\hat{m}_{k l}(u)$ follows from Theorem 1 and the fact that $E\left(\hat{p}_{k}\right)=p_{k}$.

The second-order moment follows from Theorem 1 and the relations

$$
\begin{aligned}
\operatorname{cov}\left\{\hat{p}_{k_{1}}, \hat{q}_{k_{2} l_{2}}(v)\right\}= & \frac{2 \pi H_{k_{1} k_{2} l_{2}}^{(T)}(0)}{H_{k_{1}}^{(T)}(0) H_{k_{2} l_{2}}^{(T)}(0)} \int_{-\pi / b}^{+\pi / b} f_{k_{1} k_{2} l_{2}}(0, \lambda) \exp (i \lambda v) d \lambda \\
& +O\left(T^{-2}\right)
\end{aligned}
$$

and

$$
\operatorname{cov}\left(\hat{p}_{k_{1}}, \hat{p}_{k_{2}}\right)=\frac{2 \pi H_{k_{1} k_{2}}^{(T)}(0)}{H_{k_{1}}^{(T)}(0) H_{k_{2}}^{(T)}(0)} f_{k_{1} k_{2}}(0)+O\left(T^{-2}\right)
$$

Finally, the asymptotic normality follows from the fact that the expressions

$$
\begin{aligned}
T^{J / 2} & \operatorname{cov}\left\{\hat{q}_{k_{1} l_{1}}(u), \hat{q}_{k_{2} l_{2}}(u), \ldots, \hat{q}_{k_{J} l_{j}}(u)\right\}, \\
& T^{J / 2} \operatorname{cov}\left\{\hat{p}_{k_{1}}, \hat{q}_{k_{2} l_{2}}\left(u_{2}\right), \ldots, \hat{q}_{k_{J} l_{j}}\left(u_{J}\right)\right\}, \\
& T^{J / 2} \operatorname{cov}\left\{\hat{p}_{k_{1}}, \hat{p}_{k_{2}}, \ldots, \hat{q}_{k_{J} l_{J}}\left(u_{J}\right)\right\}, \ldots, T^{J / 2} \operatorname{cov}\left(\hat{p}_{k_{1}}, \hat{p}_{k_{2}}, \ldots, \hat{p}_{k_{J}}\right)
\end{aligned}
$$

tend to zero as $T \rightarrow \infty$ and $J>2$. 
Using again Lemma 3 of Mann and Wald [12], we find

$$
\hat{\xi}_{k l}(u)-\xi_{k l}(u)=\frac{1}{2 \xi_{k l}(u)}\left\{\hat{m}_{k l}(u)-m_{k l}(u)\right\}+o_{p}\left(T^{-1}\right),
$$

provided that $\xi_{k l}(u) \neq 0$ for every $u$.

The rest of the proof follows from Theorem 2 .

\section{REFERENCES}

1 M. S. Bartlett, The spectral analysis of point processes, J. Roy. Stat. Soc. B 25:264-296 (1963).

2 P. Bloomfield, Fourier Analysis of Time Series: An Introduction, Wiley, New York, 1976.

3 I. A. Boyd, The structure and innervation of the nuclear bag muscle fibre system and the nuclear chain muscle fibre system in mammalian muscle spindles, Phil. Trans. Roy. Soc. B 245:83-136 (1962).

4 I. A. Boyd, The isolated mammalian muscle spindle, Trends Neurosci. 3:258-265 (1980).

5 D. R. Brillinger, The spectral analysis of stationary interval functions, Proc. 6th Berkeley Symposium on Mathematical Statistics and Probability, Vol. 1, Univ. California Press, Berkeley, 1972, pp. 483-513.

6 D. R. Brillinger, Estimation of product densities, Proc. Computer Science and Statistics: 8th Annual Symposium on Interface, UCLA, Los Angeles, 1975, pp. 431-438.

7 D. R. Brillinger, Estimation of second-order intensities of a bivariate stationary point process, J. Roy. Stat. Soc. B 38:60-66 (1976).

8 D. R. Brillinger, Time Series: Data Analysis and Theory, Holt, Rinehart and Winston, London, 1981.

9 D. R. Cox, On the estimation of the intensity function of a stationary point process, J. Roy. Stat. Soc. B 27:332-337 (1965).

10 D. R. Cox and P. A. W. Lewis, Multivariate point processes, Proc. 6th Berkeley Symposium on Mathematical Statistics and Probability, Vol. 2, Univ. California Press, Berkeley, 1972, pp. 401-448.

11 H. Doss, On estimating the dependence between two point processes, Ann. Stat. 14:749-763 (1989).

12 H. B. Mann and A. Wald, On stochastic limit and order relations, Ann. Math. Stat. 14:217-226 (1943).

13 P. B. C. Matthews, Review lecture: evolving views on the interval operation and functional role of the muscle spindle, J. Physiol. 320:1-30 (1981).

14 A. G. Rigas, Point processes and time series analysis: theory and applications to complex physiological problems, Ph.D. Thesis, University of Glasgow, 1983.

15 L. Torres-Melo, Stationary point processes, Ph.D. Thesis, University of California, Berkeley, 1974.

16 P. D. Tuan, Estimation of the spectral parameters of a stationary point process, Ann. Stat. 9:615-627 (1981).

17 J. W. Tukey, The estimation of power spectra and related quantities, On Numerical Approximation, Univ. Wisconsin Press, Madison, 1959, pp. 389-411. 